\title{
Estimation of Annual Health Care Costs for Adults with Type 1 Diabetes in the United States
}

\author{
Vijay N. Joish, PhD; Fang Liz Zhou, MD, PhD; Ronald Preblick, PharmD; \\ Dee Lin, PharmD, MS; Maithili Deshpande, PhD; Sumit Verma, MS; \\ Michael J. Davies, PhD; Sachin Paranjape, PhD; and Jeremy Pettus, MD
}

\begin{abstract}
BACKGROUND: Diabetes health care resource utilization (HCRU) studies tend to focus on patients with type 2 diabetes (T2D) or pool patients with T2D and type 1 diabetes (T1D). There is a paucity of recent data on the cost of treating patients with T1D in the United States.

OBJECTIVES: To (a) estimate the per-patient per-year (PPPY) HCRU and costs, from a payer perspective, associated with treating U.S. adults with T1D and (b) compare these with the HCRU and costs for patients with T2D.

METHODS: This retrospective cohort study used claims data from the Optum Clinformatics database between January 2015 and December 2017. Adults (aged $\geq 18$ years) with a diagnosis of T1D were propensity scorematched to adults with T2D. Overall and nondiabetes-related HCRU and costs were assessed for T1D and T2D and compared between the 2 groups.

RESULTS: Propensity scores were used to match 10,103 patient pairs from T1D and T2D cohorts (mean ages 54.4 and 56.9 years, respectively). In the T1D cohort, inpatient, emergency department (ED), outpatient, and prescription claims occurred in $14.0 \%, 17.3 \%, 85.5 \%$, and $100 \%$ of patients, respectively, resulting in a mean total cost of U.S. \$18,817 PPPY (diabetesrelated $=\$ 11,002$; nondiabetes-related $=\$ 7,816$ ). The T1D cohort had significantly higher mean total costs than the T2D cohort ( $\$ 18,817$ vs. $\$ 14,148$ PPPY; $P<0.001)$. When extrapolating these findings to a commercial health plan with 1 million covered lives, the estimated total direct medical costs of T1D would be $\$ 103.4$ million.
\end{abstract}

CONCLUSIONS: This study showed that the total annual cost of managing an adult with T1D is significantly higher than that of an adult with T2D. Nondiabetes costs accounted for $40 \%$ of the total per-patient cost, similar to patients with T2D, confirming that as patients with T1D live longer lives, they may also be at greater risk for cardiometabolic complications.

J Manag Care Spec Pharm. 2020;26(3):311-18

Copyright $\odot 2020$, Academy of Managed Care Pharmacy. All rights reserved.

\section{What is already known about this subject}

Approximately $5 \%$ of patients with diabetes have type 1 diabetes (T1D), over two-third of whom have difficulty achieving glycemic control.

Health care costs of managing patients with type 2 diabetes (T2D) have been well characterized

\section{What this study adds}

This study shows that patients with T1D, even though fewer in number, have significantly higher mean total medical costs per patient per year compared with patients with T2D (\$18,817 vs. \$14,148) Over $40 \%$ ( $\$ 7,816$ in T1D) of medical costs are related to care not directly related to diabetes.

T n the U.S., approximately 30 million individuals have diabetes mellitus, of whom $95 \%$ have type 2 diabetes (T2D). ${ }^{1}$ As with T2D, type 1 diabetes (T1D) carries a lifelong risk of long-term sequelae, such as cardiovascular disease and microvascular complications, as well as acute risks of ketoacidosis and hypoglycemia., ${ }^{2,3}$ However, T1D and T2D differ in terms of disease etiologies and risk factors. ${ }^{4}$ Additionally, in T1D, the cornerstone of treatment is insulin, and patients are mostly under the care of endocrinologists or other specialists, whereas uncomplicated T2D patients are managed by general practitioners primarily using oral antihyperglycemic drugs (OADs), with insulin therapy usually reserved for patients who are uncontrolled on OADs. ${ }^{5,6}$ Therefore, it is important from both public health surveillance and planning perspectives to monitor the individual prevalence of TID and associated health care resource utilization (HCRU) and costs. The number of patients with diabetes is projected to reach 39.7 million in the United States (13.9\% of the population) by 2030 and 60.6 million (17.9\% of the population) by $2060 .^{7,8}$ Although this alarming increase in prevalence is primarily due to $\mathrm{T} 2 \mathrm{D}$, the contribution of TID to the burden of care of diabetes in general is overlooked when the 2 diseases are considered together. ${ }^{9}$

There is a paucity of data directly relating to HCRU and costs for people with T1D, with most economic research combining both types of diabetes or focusing on T2D. ${ }^{9}$ Although cost estimates of TID may be extrapolated using prevalence data of T1D and T2D in the overall diabetes population, the true cost of TID is often unclear due to the larger prevalencedriven T2D costs. Existing economic data relating to T1D often lack specific relevance to the U.S. population, currency, or a suitable comparator or are specific to one aspect of the disease rather than providing economic data for the disease as a whole. ${ }^{9-14}$ 
The present analysis was designed to estimate the annual per-patient HCRU and costs (overall and nondiabetes-related) associated with the care of adults with T1D and compared to a matched T2D cohort from a commercial payer perspective. Since any comparison of diabetes-related costs between patients with T1D and T2D would be potentially misleading due to differences in management and care models (e.g., injectables and specialist management with TID vs. oral antidiabetic medications and predominantly primary care management with T2D), the present analysis focused on overall and nondiabetes-related HCRU and costs. In addition, capturing nondiabetes-related HCRU and costs helps to estimate the costs associated with managing complications.

\section{Methods}

\section{Study Design and Data Source}

This retrospective cohort study used the Optum Clinformatics database, a large integrated medical and pharmacy claims dataset of $>25$ million commercially insured and Medicare Advantage members enrolled with the UnitedHealthcare insurance provider. Patients were identified from January 1, 2016, to December 31, 2016. The index date was the date of the first medical claim for T1D or T2D during the identification period. The baseline period was the 12 months prior to the index date. Patients were then followed until 12 months following the index date or death, whichever occurred first. The Optum Clinformatics database is compliant with the Health Insurance Portability and Accountability Act of 1996. All data were deidentified prior to acquisition, and accordingly, institutional review board approval was not required nor sought. The study was conducted in accordance with ethical principles that are consistent with the Declaration of Helsinki, International Conference on Harmonization Good Clinical Practice, and any applicable local legislation.

\section{Study Population}

Patients aged $\geq 18$ years at the index date with $\geq 1$ T1D or T2D medical claims (International Classification of Diseases, Ninth Revision, Clinical Modification [ICD-9-CM] codes 250.00-250.03, 250.10-250.13, 250.20-250.23, 250.30-250.33, 250.40-250.43, 250.50-250.53, 250.60-250.63, 250.70-250.73, 250.80-250.83, and 250.90-250.93 or Tenth Revision [ICD-10-CM] codes E10.0-E10.6, E10.8, E10.9, E11.0-E11.6, E11.8, and E11.9 in the primary or secondary position) during the identification period were eligible. Patients were classified to T1D or T2D by applying a validated Klompas algorithm to the entire lookback period based on data from 2006 to 2016 in the database prior to the index date. ${ }^{15}$ The use of the Klompas algorithm to distinguish between patients with T1D and T2D overcomes the problem of inaccurate coding of diabetes type within health care records and pharmacy claims. The positive predictive value (PPV) for the Klompas algorithm was $89 \%$ in the original publication and $94.5 \%$ in an external validation study. ${ }^{15,16}$
Patients were required to have continuous enrollment during the 12-month baseline period. Patients with any of the following were excluded: a follow-up period of $<30$ days post-index date or any medical claim for pregnancy (ICD-9-CM codes 630.xx-679.xx and V22-V24 and ICD-10-CM codes O00-O9A, Z33, Z34, Z36, and Z3A) 12 months prior to index date. In order to ensure that the enrolled patients were being actively treated, the exclusion criteria in the TID cohort specified those without any claim of short-acting or rapid-acting insulin within 6 months prior to index date and, for patients in the T2D cohort, included those without any antihyperglycemic medication in the 12-month baseline period.

\section{Data Handling}

Given the large disparity in numbers of patients with T1D compared with T2D, a random sample of $10 \%$ of patients with T2D was selected before matching with patients with T1D. Propensity score nearest neighbor 1:1 matching (PSM) with a 0.2 caliper was implemented to obtain a matched sample with similar demographics for patients with T1D and T2D. ${ }^{17}$ Matching was performed using only demographic characteristics (i.e., categorical age, sex, race, and geographic region) to allow evaluation of the impact of clinical characteristics in patients with T1D and T2D by adding these as covariates in multivariate regression. The T2D cohort was used as a reference population. Patient characteristics were compared before and after propensity score matching to ensure that cohorts were matched appropriately. The analyses were carried out using only the matched cohort.

\section{Outcome Assessments}

Study outcomes included HCRU and HCRU-associated costs, which were calculated as per-patient per-year (PPPY) for the T1D and T2D cohorts. Among patients who died and had a follow-up period of less than 12 months, HCRU-associated cost was annualized by multiplying their average monthly cost by 12 . HCRU outcomes were calculated based on the number of encounters or prescriptions identified in the baseline and follow-up periods, and HCRU included inpatient visits, emergency department (ED) visits, outpatient office visits, and prescription claims. Cost calculations included expenditures for medical care and treatment of illness, with ED visits that resulted in hospitalization included in hospital care costs.

All costs were adjusted for inflation to 2017 U.S. dollars using the annual medical care component of the Consumer Price Index. Costs were defined as total standardized gross payments (not charges), including commercial insurance payments, as well as patient copayments, deductibles, and coinsurance fees. HCRU-associated costs included inpatient, ED, outpatient, pharmacy, and total costs. HCRU and cost were classified as all-cause, diabetes-related, or nondiabetes-related; claims with ICD codes for diabetes in the primary or secondary 


\section{FIGURE 1 Patient Attrition}

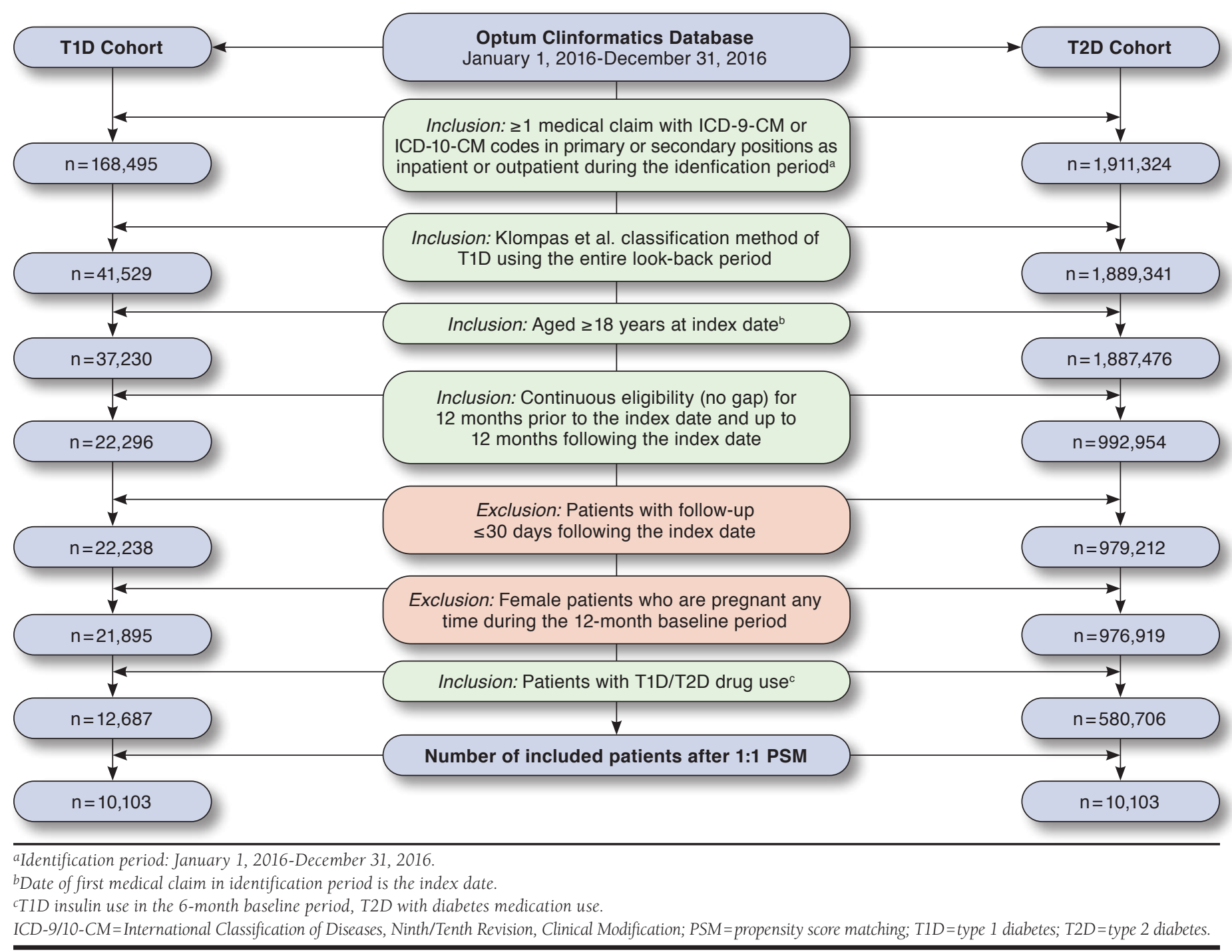

position were defined as diabetes-related; otherwise, they were defined as nondiabetes-related.

\section{Statistical Analyses}

Outcomes of interest were analyzed using descriptive and inferential statistics for individuals who were not missing demographic data. Missing data were considered as missing at random. This approach resulted in the omission of data from 3 patients for whom gender data were missing. Statistical tests were 2-sided, with a significance level of $\alpha=0.05$. Demographic characteristics, HCRU, and cost outcomes were summarized descriptively, with differences in characteristics between TlD and T2D patients assessed using chi-square tests for categorical variables and t-tests for continuous variables. In addition to $P$ values, standardized mean differences (SMDs), defined as the absolute difference in sample means divided by an estimate of the pooled standard deviation (SD) of the continuous and categorical variables, were calculated to account for the large sample size of the study, as described by Austin (2011).18

Statistically significant differences were defined when both the $P$ value was $<0.05$ and the SMD was $\geq 0.1 .{ }^{19}$ Multivariate analyses were conducted with outcomes that achieved statistical significance in univariate analyses. For HCRU count outcomes, a generalized linear model (GLM) was developed, with Poisson distribution and log link for count-dependent and variables. An offset was added to regression models to account 
TABLE 1 Demographics and Baseline Characteristics

\begin{tabular}{|c|c|c|c|c|c|c|}
\hline \multirow{2}{*}{$\begin{array}{l}\text { Characteristic } \\
\text { Age, years }\end{array}$} & \multicolumn{2}{|c|}{$\begin{array}{c}\text { T1D Cohort } \\
n=10,103\end{array}$} & \multicolumn{2}{|c|}{$\begin{array}{c}\text { T2D Cohort } \\
n=10,103\end{array}$} & \multirow{2}{*}{$\begin{array}{l}P \text { Value }^{\mathrm{a}} \\
<0.001\end{array}$} & \multirow{2}{*}{$\frac{\text { SMD }^{b}}{0.172}$} \\
\hline & & & & & & \\
\hline Mean (SD) & 54.4 & $(14.9)$ & 56.9 & $(13.8)$ & & \\
\hline Median (Q1-Q3) & 55 & $(43-66)$ & 56 & $(46-66)$ & & \\
\hline \multicolumn{5}{|c|}{ Age category, years, \% (n) } & 0.993 & 0.004 \\
\hline $18-25$ & 0.7 & $(73)$ & 0.7 & $(73)$ & & \\
\hline $26-49$ & 36.6 & $(3,696)$ & 36.5 & $(3,690)$ & & \\
\hline $50-64$ & 36.1 & $(3,648)$ & 36.3 & $(3,667)$ & & \\
\hline$\geq 65$ & 26.6 & $(2,686)$ & 26.5 & $(2,673)$ & & \\
\hline Female sex, \% (n) & 48.4 & $(4,893)$ & 49.2 & $(4,968)$ & 0.481 & 0.017 \\
\hline \multicolumn{5}{|l|}{ Race, \% (n) } & 0.219 & 0.034 \\
\hline African American & 7.2 & $(729)$ & 7.4 & $(744)$ & & \\
\hline Asian & 1.8 & $(185)$ & 1.8 & $(184)$ & & \\
\hline Hispanic & 5.2 & $(527)$ & 5.2 & $(523)$ & & \\
\hline White & 71.4 & $(7,212)$ & 70.1 & $(7,086)$ & & \\
\hline Other/unknown & 14.4 & $(1,450)$ & 15.5 & $(1,566)$ & & \\
\hline \multicolumn{5}{|l|}{ Payer type, \% (n) } & $<0.001$ & 0.088 \\
\hline Commercial & 71.9 & $(7,271)$ & 67.9 & $(6,863)$ & & \\
\hline Medicare & 28.0 & $(2,832)$ & 32.1 & $(3,240)$ & & \\
\hline \multicolumn{5}{|c|}{ Geographic region, \% (n) } & 0.004 & 0.055 \\
\hline Midwest & 28.4 & $(2,866)$ & 27.7 & $(2,803)$ & & \\
\hline South & 39.6 & $(4,004)$ & 42.2 & $(4,263)$ & & \\
\hline West & 21.8 & $(2,202)$ & 20.6 & $(2,081)$ & & \\
\hline Northeast & 10.0 & $(1,015)$ & 9.3 & $(943)$ & & \\
\hline Other/unknown & 0.2 & $(16)$ & 0.1 & $(13)$ & & \\
\hline \multicolumn{7}{|c|}{ Top 5 T2D comorbidities, ${ }^{\mathrm{c}} \%$ (n) } \\
\hline Hypertension & 57.7 & $(5,830)$ & 75.6 & $(7,639)$ & $<0.001$ & 0.387 \\
\hline Hyperlipidemia & 65.3 & $(6,595)$ & 73.2 & $(7,394)$ & $<0.001$ & 0.172 \\
\hline Obesity & 10.1 & $(1,018)$ & 27.8 & $(2,807)$ & $<0.001$ & 0.464 \\
\hline Hypothyroidism & 32.3 & $(3,266)$ & 19.0 & $(1,919)$ & $<0.001$ & 0.309 \\
\hline GERD & 13.5 & $(1,362)$ & 17.4 & $(1,761)$ & $<0.001$ & 0.109 \\
\hline \multicolumn{7}{|c|}{$\begin{array}{l}\text { a Statistically significant result defined as } P<0.05 . \\
\text { bStatistically significant result defined as } S M D \geq 0.1 \text {. } \\
\text { 'Comorbidities that are commonly observed in } T 2 D \text { patients. } \\
\text { GERD= gastroesophageal reflux disease; } Q 1=\text { first quartile; } 23=\text { third quartile; } S D=\text { standard deviation; } S M D=\text { standardized mean difference; T1D = type } 1 \text { diabetes } \\
T 2 D=\text { type } 2 \text { diabetes. }\end{array}$} \\
\hline
\end{tabular}

for differences in follow-up periods between patients, which ranged from 30 days to 12 months. Health care costs were analyzed using a 2-part model, with logistic regression for the binomial component and standard ordinary least squares for the continuous component. In the 2-part model, the probability of positive cost was estimated with logistic regression and conditional on the cost being positive, and the value of positive cost was modeled with standard ordinary least squares. Given the structure of the 2-part model, each model was fit separately, and the predictions were combined to derive mean predicted cost. All GLM and cost outcomes were adjusted for demographic and clinical characteristics that were found to be significantly different between matched T1D and T2D cohorts. T2D results were used as reference values.

\section{Results}

\section{Patient Attrition and Baseline Characteristics}

A total of 12,687 patients with T1D and 580,706 patients with T2D met all eligibility criteria (Figure 1). Of these, 10,103 patient pairs in the T1D and T2D patient cohorts were 1:1 propensity score-matched for further analyses. Patients in the T1D and T2D cohorts were generally well matched in terms of baseline demographics and clinical characteristics (Table 1). Although age groups were well balanced in the 2 cohorts, the mean (SD) age was lower in the T1D cohort compared with the T2D cohort (54.4 [14.9] vs. 56.9 [13.8] years; $P<0.001 ;$ SMD $>0.1$ ). Median (Q1-Q3) age was 55.0 (43-66) compared with 56.0 (46-66), respectively. The difference in age was accounted for in the multivariate model. Patients with T1D versus T2D were significantly less likely to have diagnoses of hypertension $(57.7 \%$ 


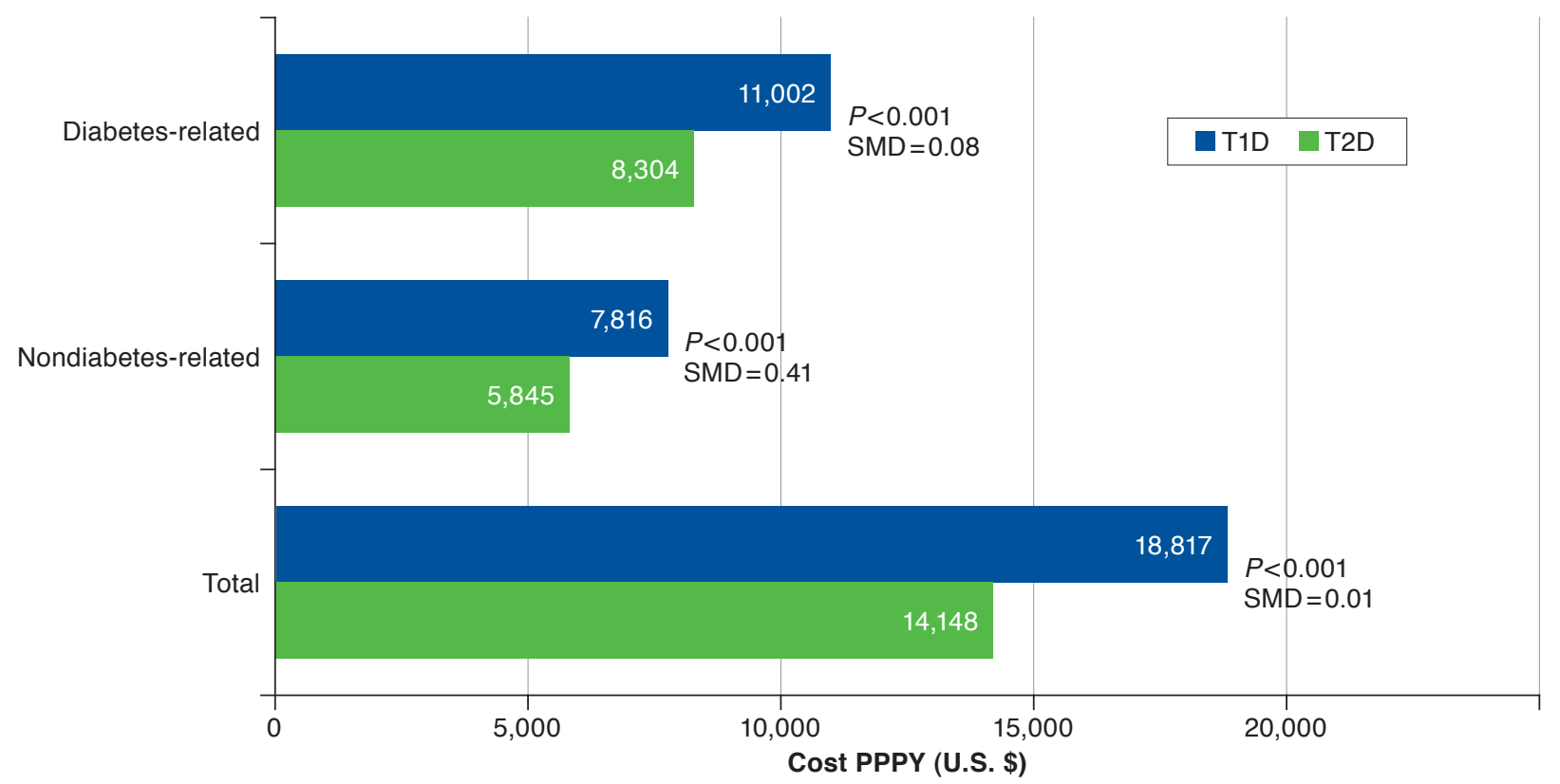

Note: Data were adjusted for differences in age, comorbidity index, resource use, and top 5 comorbidities (hypertension, hyperlipidemia, hypothyroidism, obesity, and gastroesophageal reflux disease).

$P P P Y=$ per person per year; SMD = standardized mean difference; $T 1 D=$ type 1 diabetes; $T 2 D=$ type 2 diabetes; U.S. =United States.

vs. $75.6 \%)$, hyperlipidemia (65.3\% vs. $73.2 \%)$, obesity (10.1\% vs. $27.8 \%$ ), and gastroesophageal reflux disease (13.5\% vs. 17.4\%) and were more likely to have hypothyroidism (32.3\% vs. $19.0 \%$ ) (all, $P<0.001$ and SMD $>0.1$ ).

\section{Health Care Resource Utilization and Costs}

Patients with TID had significantly higher mean total costs PPPY compared with patients with T2D $(\$ 18,817[37,661]$ vs. $\$ 14,148[46,555] ; P<0.001 ; S M D=0.110)$. The median total cost PPPY was $\$ 10,404$ compared with $\$ 5,262$, respectively. The average total cost to the payer of a TlD patient was estimated to be $\$ 18,817$ PPPY, $42 \%(\$ 7,816)$ of which were costs not directly related to the management of diabetes (Figure 2).

In comparison, the average total cost to the payer of a T2D patient was estimated to be $\$ 14,148$ PPPY, $41 \%(\$ 5,845)$ of which were costs not directly related to the management of diabetes. Mean (SD) total prescription costs PPPY were significantly higher for patients with T1D compared with T2D $(\$ 10,203[11,211]$ vs. $\$ 5,672[11,017] ; P<0.001 ; S M D=0.408$; respectively), as were mean diabetes-related $(\$ 4,955[3,428]$ vs. $\$ 2,223[3,888] ; P<0.001 ; \mathrm{SMD}=0.745)$ and nondiabetesrelated prescription costs $(\$ 5,247[10,492]$ vs. $\$ 3,449[10,010]$; $P<0.001$; SMD $=0.175$ ). Median total prescription costs PPPY were $\$ 7,816$ and $\$ 2,783$ for the T1D and T2D cohorts, respectively. Median diabetes-related prescription costs were $\$ 4,221$ and $\$ 203$, respectively. Median nondiabetes-related prescription costs were $\$ 2,899$ and $\$ 1,055$, respectively. Costs associated with inpatient, ED, and outpatient visits were comparable between the 2 cohorts.

The proportions of T1D patients with any-cause inpatient visits, ED visits, outpatient visits, and prescription claims were $14.0 \%(n=1,416), 17.3 \%(n=1,744), 85.5 \%(n=8,643)$, and $100 \%(n=10,103)$, respectively (Figure 3). For the T1D cohort, the average (SD) number of inpatient visits, ED visits, outpatient visits, and prescription claims was 0.3 (1.2), 1.0 (3.8), 5.5 (5.8), and 45.0 (35.7) PPPY, respectively. The respective median values for number of inpatient, ED, and outpatient visits and prescription claims was $0,0,4$, and 36 .

In comparison, $13.1 \%(n=1,322), 16.7 \%(n=1,689), 83.0 \%$ $(n=8,388)$, and $100 \%(n=10,103)$ of the T2D cohort had anycause inpatient visits, ED visits, outpatient visits, and prescription claims, respectively, with an average (SD) number of 0.3 (1.2), 0.9 (3.4), 5.0 (5.6), and 49.1 (37.9) inpatient visits, ED visits, outpatient visits, and prescription claims PPPY, respectively. The respective median values were $0,0,4$, and 40 . When comparing between the 2 cohorts, patients with T1D compared with T2D had a significantly higher mean number of any-cause outpatient visits (5.5 vs. 5.0; $P<0.001 ; \mathrm{SMD}=0.085$ ) and a significantly lower mean number of any-cause prescription claims (45.0 vs. $49.1 ; P<0.001 ; \mathrm{SMD}=0.112$ ). 


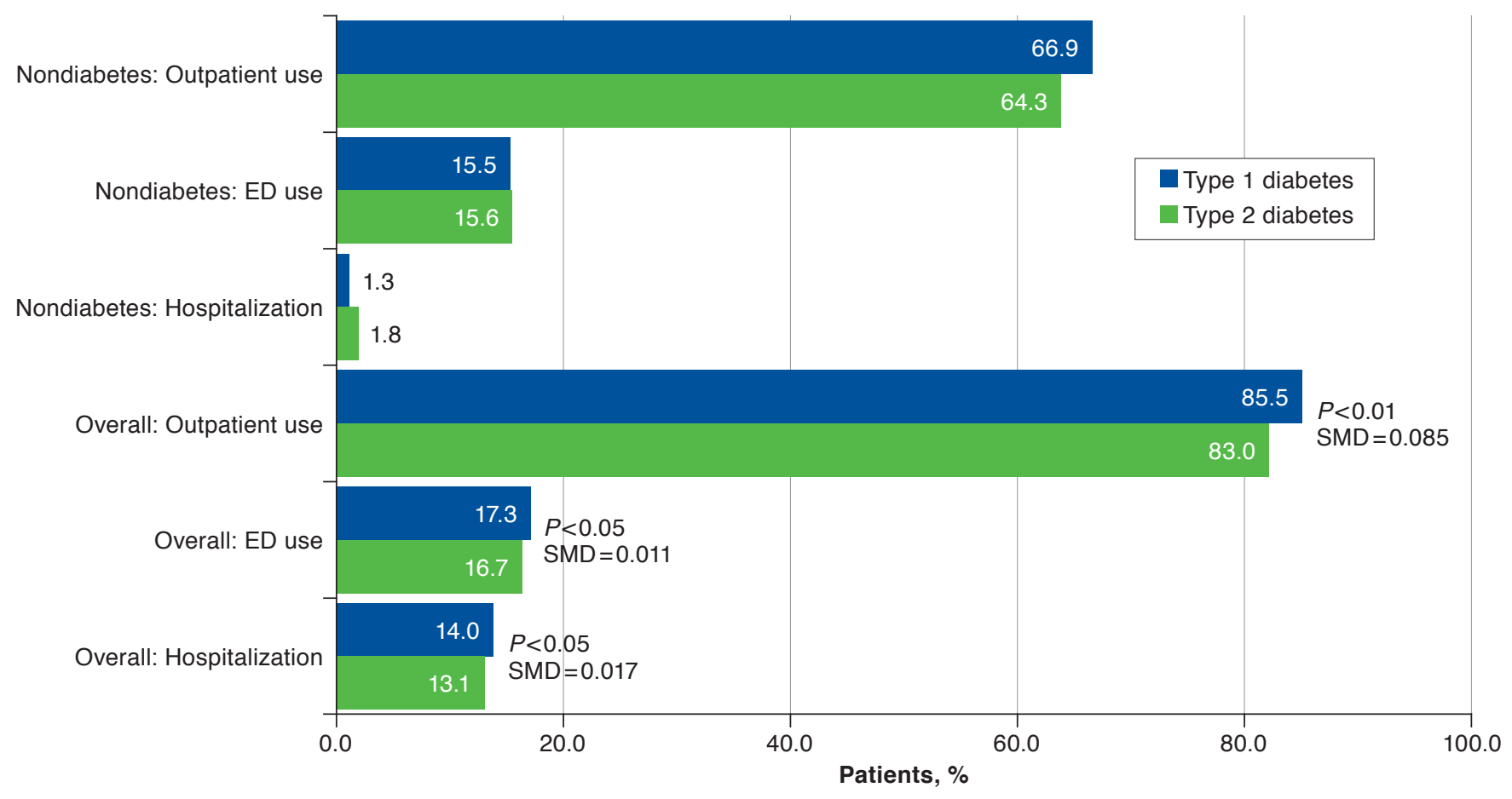

Note: Data were adjusted for differences in age, comorbidity index, resource use, and top 5 comorbidities (hypertension, hyperlipidemia, hypothyroidism, obesity, and gastroesophageal reflux disease).

$D M=$ diabetes mellitus; ED=emergency department.

When considering nondiabetes-related resource utilization, the proportions of T1D patients with inpatient visits, ED visits, outpatient visits, and prescription claims were 1.3\% $(n=129), \quad 15.5 \%(n=1,568), 66.9 \%(n=6,762)$, and $99.2 \%$ $(\mathrm{n}=10,027)$, respectively (Figure 3$)$. The average (SD) number of outpatient visits and prescription claims was 2.9 (4.1) and 36.7 (32.9) PPPY, respectively. In the cohort of T2D patients, $1.8 \%(n=179), 15.6 \%(n=1,580), 64.3 \%(n=6,500)$, and $99.2 \%$ $(\mathrm{n}=10,020)$ had nondiabetes-related inpatient visits, ED visits, outpatient visits, and prescription claims, respectively. The average (SD) number of outpatient visits and prescription claims PPPY was 2.9 (4.4) and 38.6 (34.6), respectively. Overall inpatient, ED, and outpatient HCRU were similar for patients with T1D and T2D.

\section{Discussion}

The mean annual cost to the payer for managing the care of an adult with T1D was estimated to be $\$ 18,817$ (in 2016-2017), which was significantly higher than for T2D $(\$ 14,148)$. Further, approximately $40 \%$ of medical costs were nondiabetes-related for patients with T1D or T2D. When extrapolating these findings to a commercial health plan with 1 million covered lives, the estimated overall annual total direct medical cost burden of adults with TID is estimated to be U.S. \$103.4 million.

Overall, drivers of higher utilization and costs among patients with T1D versus patients with T2D included outpatient all-cause diabetes-related physician visits, prescription costs, and outpatient costs. Given that patients with TID are treated (e.g., insulin vs. oral antidiabetes prescriptions) and managed (e.g.. endocrinologists vs. primary care physicians) differently versus patients with T2D, we also compared nondiabetes-related health care utilization and costs. Drivers of nondiabetes-related utilization and costs among patients with T1D versus patients with T2D also included greater outpatient services and costs and prescription claims and costs. This may suggest that even though both types of diabetes are lifelong conditions, given the earlier onset of disease and higher proportion of patients not reaching glycemic goals, patients with T1D at a given point in time may have higher morbidity, resulting in higher health care resource consumption and cost to the system. Improving glycemic control rates by increasing patient education and providing access to cost-effective treatments and 
technology may help in improving both health and economic outcomes in this population.

Willey et al. (2018) examined diabetes costs in the United States. ${ }^{8}$ Using health care encounters with codes for diabetes or antihyperglycemic medications, the estimated mean T1Drelated PPPY cost was $\$ 7,122$ in 2014 . This is generally similar to the diabetes-related costs in the current analysis $(\$ 11,001)$. Similar to our study, Willey et al. ${ }^{8}$ also reported that patients with T1D had higher costs than those with T2D. ${ }^{7}$

\section{Limitations}

The results of the current analysis should be interpreted with consideration of certain strengths and limitations. Although use of a single-payer database may limit the generalizability of these results, availability and use of a multiplan database from one of the largest national insurers using a large sample may be well accepted by other payers as a starting point. Data coding errors, which are not uncommon in such databases, cannot be fully avoided or robustly identified without access to the primary source data. However, this may not be a concern since the estimation of the burden of disease is based on paid claims and reflective of a payer perspective. Missing data were assumed to be missing at random, but this may not have been the case. Misclassification bias, which is another common issue when using a nonclinical administrative claims database, was addressed 2-fold, using a validated and published TID and T2D classification algorithm with very high sensitivity and PPVs and requiring patients with T1D to have prescription claims for bolus insulin 6 months prior to the index date. ${ }^{15}$

Although PSM was used to minimize any differences between TID and T2D patients, there remained the potential for bias. In particular, matching patients on age may have led to the inclusion of TID patients who, by virtue of being diagnosed with their disease at a younger age, had been living with diabetes for much longer than their age-matched T2D counterparts, resulting in higher associated cost estimates. However, a recent publication by Rogers et al. (2017) estimated that of the 64,000 incident cases of T1D in the United States, 37,000 of these (58\%) were adult-onset cases, suggesting that it was appropriate in our study to match patients with T1D and T2D according to age..$^{20}$

\section{Conclusions}

The mean annual total cost to the payer for managing the care of adults with T1D amounts to approximately \$18,817 PPPY, with around $40 \%$ of this cost associated with nondiabetesrelated claims. ${ }^{7,15,17}$

\section{Authors}

VIJAY N. JOISH, PhD, and MICHAEL J. DAVIES, PhD, Lexicon Pharmaceuticals, The Woodlands, Texas. FANG LIZ ZHOU, MD; RONALD PREBLICK, PharmD; DEE LIN, PharmD, MS; and SACHIN PARANJAPE, PhD, Sanofi, Bridgewater, New Jersey. MAITHILI DESHPANDE, PhD, Southern Illinois University, Edwardsville; SUMIT VERMA, PhD, Evidera, Bethesda, Maryland; and JEREMY PETTUS, MD, University of California, San Diego.

AUTHOR CORRESPONDENCE: Vijay N. Joish, PhD, Lexicon Pharmaceuticals, 8800 Technology Forest Pl., The Woodlands, TX 77381. Tel: 908.360.4782; E-mail: vjoish@LexPharma.com.

\section{DISCLOSURES}

This study was funded by Sanofi U.S. and Lexicon Pharmaceuticals as part of a business partnership in a diabetes program at the time this study was conducted. Joish and Davies are employees and stockholders of Lexicon Pharmaceuticals. Zhou, Preblick, and Paranjape are employees and stockholders of Sanofi. Lin was a postdoctoral fellow at Sanofi through Rutgers University during this project. Deshpande provided consulting services through Communication Symmetry. Verma is an employee of Evidera, which was contracted by Sanofi for work on this study. Pettus is a consultant for Diasome, Insulet, Lexicon, Lilly, Mannkind, Novo Nordisk, Sanofi, and Senseonics.

\section{ACKNOWLEDGMENTS}

The authors received writing/editorial support in the preparation of this manuscript from Joseph Worrall, PhD, of Excerpta Medica, funded by Sanofi U.S.

\section{REFERENCES}

1. Centers for Disease Control and Prevention. National Diabetes Statistics Report, 2017. Available at: https://www.cdc.gov/diabetes/pdfs/data/statistics/ national-diabetes-statistics-report.pdf. Accessed February 10, 2020

2. Fullerton B, Jeitler K, Seitz M, Horvath K, Berghold A, Siebenhofer A Intensive glucose control versus conventional glucose control for type 1 diabetes mellitus. Cochrane Database Syst Rev. 2014;(2):CD009122.

3. Smith MJ, Simmons KM, Cambier JC. B cells in type 1 diabetes mellitus and diabetic kidney disease. Nat Rev Nephrol. 2017;13(11):712-20.

4. Zaccardi F, Webb DR, Yates T, Davies MJ. Pathophysiology of type 1 and type 2 diabetes mellitus: a 90-year perspective. Postgrad Med J. 2016;92(1084):63-69.

5. Jenum AK, Claudi T, Cooper JG. Primary care diabetes in Norway. Prim Care Diabetes. 2008;2(4):203-05.

6. Honkasalo MT, Linna M, Sane T, Honkasalo A, Elonheimo O. A comparative study of two various models of organising diabetes follow-up in public primary health care-the model influences the use of services, their quality and costs. BMC Health Serv Res. 2014;14:26.

7. Lin J, Thompson TJ, Cheng YJ, et al. Projection of the future diabetes burden in the United States through 2060. Popul Health Metr. 2018;16(1):9.

8. Willey VJ, Kong S, Wu B, et al. Estimating the real-world cost of diabetes mellitus in the United States during an 8-year period using 2 cost methodologies. Am Health Drug Benefits. 2018;11(6):310-18.

9. Tao B, Pietropaolo M, Atkinson M, Schatz D, Taylor D. Estimating the cost of type 1 diabetes in the U.S.: a propensity score matching method. PLoS One. 2010;5(7):el1501. 
10. Hex N, Bartlett C, Wright D, Taylor M, Varley D. Estimating the current and future costs of type 1 and type 2 diabetes in the UK, including direct health costs and indirect societal and productivity costs. Diabet Med. 2012;29(7):855-62.

11. Giorda CB, Rossi MC, Ozzello O, et al. Healthcare resource use, direct and indirect costs of hypoglycemia in type 1 and type 2 diabetes, and nationwide projections. Results of the HYPOS-1 study. Nutr Metab Cardiovasc Dis. 2017;27(3):209-16.

12. Johnson JA, Pohar SL, Majumdar SR. Health care use and costs in the decade after identification of type 1 and type 2 diabetes: a population-based study. Diabetes Care. 2006;29(11):2403-08.

13. Dall TM, Mann SE, Zhang Y, et al. Distinguishing the economic costs associated with type 1 and type 2 diabetes. Popul Health Manag. 2009;12(2):103-10

14. Fitch K, Weisman T, Engel T, et al. Longitudinal commercial claimsbased cost analysis of diabetic retinopathy screening pattterns. Am Health Drug Benefits. 2015;8(6):300-08.
15. Klompas M, Eggleston E, McVetta J, Lazarus R, Li L, Platt R. Automated detection and classification of type 1 versus type 2 diabetes using electronic health record data. Diabetes Care. 2013;36(4):914-21.

16. Schroeder EB, Donahoo WT, Goodrich GK, Raebel MA. Validation of an algorithm for identifying type 1 diabetes in adults based on electronic health record data. Pharmacoepidemiol Drug Saf. 2018;27(10):1053-59.

17. Rosenbaum PR, Rubin DB. The central role of the propensity score in observational studies for causal effect. Biometrika. 1983;70(1):41-55.

18. Austin PC. An introduction to propensity score methods for reducing the effects of confounding in observational studies. Multivariate Behav Res. 2011;46(3):399-424.

19. Austin PC. Using the standardized difference to compare the prevalence of a binary variable between two groups in observational research. Comm Stat Simul Comput. 2009;38(6):1228-34.

20. Rogers MAM, Kim C, Banerjee T, Lee JM. Fluctuations in the incidence of type 1 diabetes in the United States from 2001 to 2015: a longitudinal study. BMC Med. 2017;15:199. 